\title{
Fatores de risco para o adoecimento dos cuidadores/familiares de pacientes com consequência do acidente vascular encefálico
}

\author{
Risk factors for the illness of caregivers/familys of patients as a consequence of brain vascular \\ accident \\ Factores de riesgo para la enfermedad de los cuidadores/familiares de los pacientes a consecuencia \\ del accidente vascular cerebral
}

Amanda Oliveira Bernardino Cavalcanti de Albuquerque ORCID: https://orcid.org/0000-0002-1011-8964 Universidade de Pernambuco, Brasil E-mail: amandaobernardino@ hotmail.com

Carlos Antonio de Lima Filho ORCID: https://orcid.org/0000-0001-5517-0347 Universidade Federal de Pernambuco, Brasil E-mail: cttoni2000@gmail.com

Matheus Vinicius Barbosa da Silva ORCID: https://orcid.org/0000-0003-1295-6301

Universidade Federal de Pernambuco, Brasil E-mail: matheushue30@gmail.com

Geysiane Kelle Alves do Nascimento ORCID: https://orcid.org/0000-0002-5387-5682

Universidade Federal de Pernambuco, Brasil E-mail: geysianealves2018@gmail.com

Gilmara Aparecida da Silva Melo ORCID: https://orcid.org/0000-0003-2627-087X Universidade Federal de Pernambuco, Brasil E-mail: gilsam377@gmail.com

Rita de Cássia Santos do Nascimento ORCID: https://orcid.org/0000-0002-2712-5415 Universidade Federal de Pernambuco, Brasil E-mail: rita.santosnascimento@ufpe.br

Denilson de Oliveira Silva Junior ORCID: https://orcid.org/0000-0001-8176-3074 Escola Tecnica de Enfermagem Israel, Brasil E-mail: denilsonoliveirasilvajunior@gmail.com João Henrique de Paula Silva ORCID: https://orcid.org/0000-0002-2285-378X Faculdade Integrada Tiradentes, Brasil E-mail: jh536315@gmail.com

Shirley Bandeira de Melo

ORCID: https://orcid.org/0000-0001-7544-8326 Faculdade Integrada Tiradentes, Brasil E-mail: shirlay.bandeira@hotmail.com

Camila Nogueira Bezerra ORCID: https://orcid.org/0000-0001-7075-9467 Secretaria Estadual de Saúde de Pernambuco

E-mail: c.nogueirabezerra@ hotmail.com

Edilma de Fátima Cardoso de Lima ORCID: https://orcid.org/0000-0002-9290-161X Faculdade Integrada Tiradentes, Brasil

E-mail: edilmacardoso2008@hotmail.com

Amelino Ventura Oliveira Lima ORCID: https://orcid.org/0000-0002-7892-8971 Faculdade Integrada Tiradentes, Brasil E-mail: amelino.ventura@ soufits.com.br

Anderson Emanuel da Silva Lima ORCID: https://orcid.org/0000-0002-9455-9045

Universidade Federal de Pernambuco, Brasil E-mail: Anderson.55silva@hotmail.com

Debora Campos Moreira

ORCID: https://orcid.org/0000-0003-3394-051X Faculdade Integrada Tiradentes, Brasil E-mail: debboracm@icloud.com 


\title{
Barbara Maranhão Calábria Cavalcanti \\ ORCID: https://orcid.org/0000-0002-1733-1071 \\ Universidade de Pernambuco, Brasil
}

E-mail: barbaracalabria.bmcc@gmail.com

\begin{abstract}
Resumo
Introdução: Pessoas acometidas por Acidente Vascular Encefálico evoluem de forma incapacitante, necessitando de um cuidador/familiar para atender às suas necessidades básicas. Objetivo: Analisar as evidências científicas sobre os principais fatores de risco para o adoecimento dos cuidadores/familiares de pacientes com Acidente Vascular Encefálico. Metodologia: Estudo realizado por meio de uma revisão integrativa, através de buscas em bases de dados eletrônicas. A análise foi descritiva. O número amostral foi de seis artigos científicos. Resultados: Quanto maior o grau de dependência, maior o desgaste físico; esgotamento emocional e que a ausência de conhecimento sobre a comorbidade e como cuidar é um fator estressante e de insegurança para o cuidador/familiar. Evidencia-se que é de suma importância que ao receber alta, estes cuidadores/familiares recebam orientação para lidar com a nova rotina do paciente. Conclusão: Os sentimentos negativos podem ser atenuados para estes cuidadores/familiares com o revezamento de outros familiares, e com apoio de profissionais da saúde na assistência ao paciente.
\end{abstract}

Palavras-chave: Acidente vascular cerebral; Cuidadores; Fatores de risco; Pacientes domiciliares.

\begin{abstract}
Introduction: People with stroke develop in a disabling way, requiring a caregiver/family member to meet their basic needs. Objective: To analyze the scientific evidence on the main risk factors for the illness of caregivers/family members of patients with stroke. Methodology: Study carried out through an integrative review, through searches in electronic databases. The analysis was descriptive. The sample number was six scientific articles. Results: The greater the degree of dependence, the greater the physical exhaustion; emotional exhaustion and that the lack of knowledge about the comorbidity and how to care is a stressful and insecure factor for the caregiver/family member. It is evident that it is of paramount importance that upon being discharged, these caregivers/family members receive guidance to deal with the patient's new routine. Conclusion: Negative feelings can be mitigated for these caregivers/family members with the rotation of other family members, and with the support of health professionals in patient care.
\end{abstract}

Keywords: Stroke; Caregivers; Risk factors; Homebound persons.

\section{Resumen}

Introducción: Las personas con ictus se desarrollan de forma discapacitante, requiriendo de un cuidador/familiar para cubrir sus necesidades básicas. Objetivo: Analizar la evidencia científica sobre los principales factores de riesgo de enfermedad de los cuidadores/familiares de pacientes con ictus. Metodología: Estudio realizado a través de una revisión integradora, a través de búsquedas en bases de datos electrónicas. El análisis fue descriptivo. El número de muestras fue de seis artículos científicos. Resultados: A mayor grado de dependencia, mayor esfuerzo físico; agotamiento emocional y que la falta de conocimiento sobre la comorbilidad y cómo cuidar es un factor estresante e inseguro para el cuidador/familiar. Es evidente que es de suma importancia que al ser dados de alta, estos cuidadores/familiares reciban orientación para enfrentar la nueva rutina del paciente. Conclusión: Los sentimientos negativos de estos cuidadores/familiares pueden ser mitigados con la rotación de otros familiares y con el apoyo de los profesionales de la salud en el cuidado del paciente.

Palabras clave: Accidente cerebrovascular; Cuidadores; Factores de riesgo; Personas imposibilitadas.

\section{Introdução}

O envelhecimento da população brasileira vem sendo considerado um dos maiores desafios para a saúde pública dos dias correntes. Estima-se que em 2021 a população idosa do território nacional era de 37,7 milhões. Onde, o crescimento desse grupo populacional em 5 anos, corresponde a uma elevação de 18\% de idosos, tornando-os uma população mais representativa ao passar de cada ano, a população feminina, dentro desse grupo etário possui destaque por ser a maioria representando $56 \%$ dos idosos, enquanto a população masculina reflete apenas 44\% dessa faixa etária (IBGE, 2018).

Ressaltando esse crescimento progressivo no quantitativo de idosos em território nacional, o Instituto Brasileiro de Geografia e Estatística divulgou que esses alcançaram a marca dos 30,2 milhões de idosos em 2017 (IBGE, 2018).

Em paralelo às modificações populacionais na qual acarreta mudanças na pirâmide populacional, patologias que acabam vindo junto com o processo fisiológico do envelhecimento, acabam se destacando, que são as doenças crônicas não transmissíveis e/ou degenerativas, como: hipertensão arterial, diabetes mellitus tipo 2, parkinson, alzheimer e dentre estas, tem-se o Acidente Vascular Encefálico (AVE), a mais impactante delas, visto que, seus sobreviventes exibem geralmente deficiências neurológicas e incapacidades residuais significativas (Vieira et al, 2011). 
Estatísticas brasileiras indicam que a AVE é a causa mais frequente de óbito na população adulta (10\% dos óbitos) e consiste no diagnóstico de 10\% das internações hospitalares públicas. Segundo Costa et al. (2021) o Brasil apresenta uma alta taxa de mortalidade por AVE em comparação com outros países da América Latina e Caribe.

Considerada a doença mais incapacitante funcional do mundo ocidental o AVE pode ser dividido em duas categorias principais: Acidente Vascular Encefálico Isquêmico (AVEI), que ocorrem em 85\% dos casos, nos quais há uma oclusão vascular e uma hipofunção significativa, e o Acidente Vascular Encefálico Hemorrágico (AVEH), que acomete em média 15\% das pessoas, quando há extravasamento de sangue para o cérebro ou para o espaço subaracnóideo (Smeltzer et al., 2005).

Para Falcão et al. (2004) ambas as categorias podem levar o indivíduo acometido a sérias consequências, fazendo com que este acabe necessitando de mais serviços de saúde, em decorrência das sequelas físicas, de comunicação e linguagem, funcionais e emocionais.

Diante disso, tem-se que os sobreviventes de AVE retornam para o domicílio necessitando de cuidados especiais e orientações para se adaptarem ao novo estilo de vida devido às limitações impostas pela doença (Pereira et al., 2013).

Desta forma a transição do cuidado hospitalar para o domicílio é um momento difícil, considerando que a perspectiva do cuidado se modifica. Para Cameron et al. (2016) no ambiente familiar a pessoa deixa de receber uma assistência especializada e passa a ser assistido por um familiar inserido em um contexto social.

De acordo com grau de comprometimento, o paciente acometido necessitará de cuidados frequentes e nesse momento, surge à figura do cuidador que na maioria dos casos é um membro da família. Porém, este familiar que passa a ser cuidador, acaba encontrando problemas, levando-o a prestar os cuidados ao paciente com consequências de AVE sem a devida capacitação para executar a mesma (Floriano et al., 2012).

Desta maneira, o despreparo no cuidar, que são ligadas ao fato de não receberem orientação e suporte dos profissionais da saúde e também da sociedade, acaba os expondo a elevada carga estressora associada a sobrecarga de trabalho, podendo afetar a saúde deste cuidador/familiar, assim como sua qualidade de vida (Israel et al., 2011).

Ressalta-se, que devido à mudança no objetivo da relação que passa a ser dependente onde anteriormente era baseada na reciprocidade, faz com que o ato de cuidar do paciente com sequela de AVE seja vista como uma atividade exaustiva e estressante (Oliveira et al., 2011).

Segundo Pereira et al. (2013), a tensão vivida pelos cuidadores/familiares eleva o risco de adquirirem doenças cardiovasculares e risco de mortalidade em $23 \%$ e $63 \%$ respectivamente, quando comparados a outro membro da família não cuidador.

Esses impactos negativos sobre a vida do cuidador/familiar podem resultar no rompimento dos cuidados prestados aos pacientes vítimas de AVE e na institucionalização desses pacientes (Cameron et al., 2016).

Na medida em que o cuidador/familiar desempenha um papel fundamental na vida do paciente vitima de AVE, ele tem a necessidade e precisa manter o seu bem-estar e uma vida saudável, recebendo cuidados e orientações em saúde, para dessa forma desenvolver a atividade do cuidado ao sequelado de AVE sem prejuízos a sua saúde. Pois, devido ao foco do cuidado ser grande, esse cuidador/familiar pode acabar negligenciando o seu próprio autocuidado (Cavalcanti et al., 2013).

A Atenção Primária à Saúde e a Atenção Domiciliar também são importantes, pois as mesmas além de atuação de interlocução entre os pacientes e hospitais, oferecem auxílio aos pacientes e também a seus cuidadores/familiares (Hoepers et al., 2019).

Sendo assim, descrever os sentimentos e emoções e os fatores que podem influenciar negativamente na vida destes cuidadores/familiares, torna-se relevante, que o profissional de saúde, conheça as necessidades do cuidador/familiar e trace metas e implemente ações no sentido de minimizar os sentimentos negativos do processo do cuidar desse cuidador/familiar.

Diante disso, este artigo tem como objetivo analisar as evidências científicas sobre os principais fatores de riscos para 
o adoecimento dos cuidadores/familiares de pacientes com AVE. A seguinte questão foi utilizada como pergunta condutora da pesquisa "Qual os principais fatores de riscos para o adoecimento dos cuidadores/familiares de pacientes com AVE?"

\section{Metodologia}

Trata-se de uma revisão integrativa da literatura, na qual tem-se que é a mais ampla abordagem metodológica, pois permite a inclusão de estudos teóricos e empíricos (Sousa et al., 2017 \& Souza et al., 2010). É uma ferramenta ímpar, pois sintetiza as pesquisas disponíveis sobre o tema e direciona o pesquisador, fundamentando-se em conhecimentos científicos (Brevidelli et al., 2008).

As etapas para a construção desse estudo foram:

Primeiramente foi delimitada a questão de pesquisa: Quais são as emoções dos cuidadores e familiares dos pacientes com sequelas do AVE?

Posteriormente foram definidos os descritores que poderiam surgir em estudos que responderiam à questão de pesquisa, os seguintes: "Assistência Domiciliar"; "Emoções"; “Cuidadores”; e "Acidente Vascular Encefálico", com o operador lógico booleano “AND”, no qual, o cruzamento dos descritores foi realizado de acordo com o Quadro 1.

A busca das literaturas para compor esse estudo foi retirada de bases de dados eletrônicas, incluindo: Bases de Dados de Enfermagem (BDENF), Literatura Latino-Americana e do Caribe em Ciências da Saúde (LILACS) e Medical Literature Analysis And Retrieval System Online (MEDLINE).

Consideraram-se ainda como critérios de inclusão adicionais: 1) artigos disponíveis na íntegra; 2) artigos no idioma português e inglês; 3) artigos publicados no período de 2014 a 2018

Já os de exclusão foram: trabalhos de conclusão de curso, dissertação, monografia e teses e aqueles fora do prazo temporal e incompletos.

Foi realizada a construção de um instrumento para a coleta de dados, devido ao elevado quantitativo de artigos encontrados sobre o assunto (43), servindo para categorizá-los, sintetizar os resultados e assim, alcançar uma melhor compreensão de cada artigo. Este foi composto por: título, ano, país, método, base de dados e principais resultados. A análise dos artigos foi realizada duplamente através de estatística descritiva.

Foi realizado o cruzamento dos descritores, conforme o Quadro abaixo:

Quadro 1. Cruzamento dos descritores sem os critérios de inclusão e com os critérios de inclusão.

\begin{tabular}{|c|c|c|}
\hline CRUZAMENTO & SEM CRITÉRIOS DE INCLUSÃO & COM CRITÉRIOS DE INCLUSÃO \\
\hline Acidente Vascular Encefálico + Cuidadores + Emoções & 0 & 0 \\
\hline $\begin{array}{c}\text { Acidente Vascular Encefálico + Cuidadores + } \\
\text { Assistência Domiciliar }\end{array}$ & 355 & 14 \\
\hline Acidente Vascular Encefálico + Cuidadores & 1.000 & 20 \\
\hline Acidente Vascular Encefálico + Assistência Domiciliar & 1.637 & 0 \\
\hline $\begin{array}{c}\text { Acidente Vascular Encefálico + Emoções + Assistência } \\
\text { Domiciliar }\end{array}$ & 11 & 0 \\
\hline Acidente Vascular Encefálico + Emoções & 203 & $\mathbf{3 . 2 0 6}$ \\
\hline TOTAL & & 43 \\
\hline
\end{tabular}

Fontes: Autores (2022). 
Na primeira busca, foram encontrados 3.206 artigos. Após selecionar aqueles que estavam em consonância com os critérios de inclusão previamente mencionados, a partir da leitura dos títulos e resumos, o total obtido foi de 43 títulos, após leitura dos artigos na íntegra, chegou-se ao total de 06 artigos, por apresentar resposta à pergunta condutora do estudo, que compõem a amostra deste estudo, sendo: dois da LILACS, um da Base de Dados BDENF e três da MEDLINE, de acordo com a Figura 1.

Salienta-se que o preenchimento do instrumento de coleta de dados foi realizado pelos autores, para que assim pudessem ser extraídos de forma eficaz os principais aspectos a serem abordados. Para a interpretação das informações a serem colhidas dos resultados, foi realizada uma leitura comparativa entre os títulos, realizando a verificação de suas similaridades e procedendose ao agrupamento.

Figura 1. Sequência da Busca na Literatura.

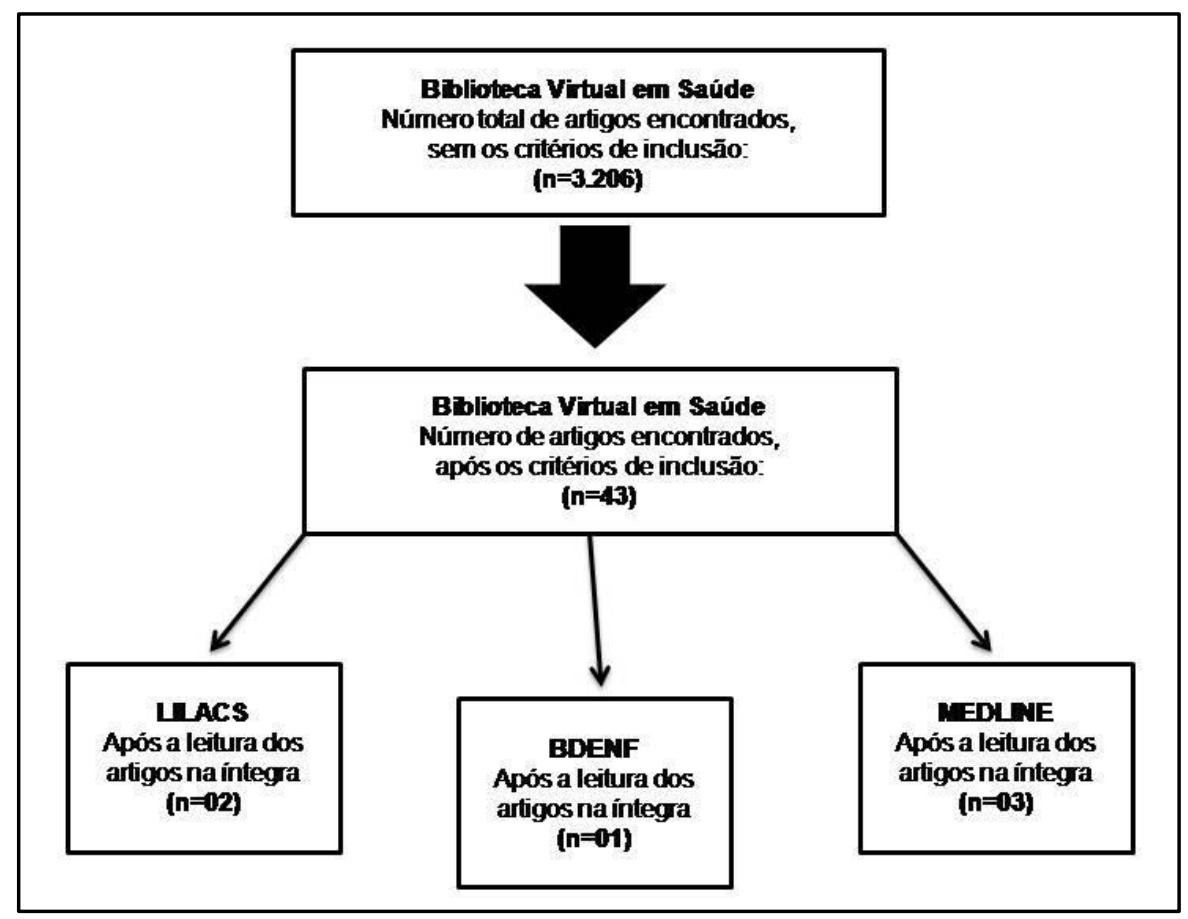

Fontes: Autores (2022).

Após a seleção dos estudos, os mesmos foram analisados de maneira sistemática e minuciosa, posteriormente, foi realizada caracterização dos estudos de acordo com Mendes et al. (2008).

\section{Resultados}

Do total de seis artigos incluídos, o método de estudo de pesquisa quantitativa foi unânime com seis títulos (100\%)

Após a leitura dos textos ficou evidente que eles não apresentam muitas diferenças entre si, sendo os achados semelhantes. Questões como depressão, sobrecarga física, cognitiva e emocional e falta de recursos financeiros e a deficiência de conhecimento acerca da doença e suas consequências, foram as questões que estiveram em mais evidência. Quanto mais o grau de dependência do paciente, mais dependente ela fica, aumentando assim os sentimentos e dificuldades do seu cuidador/familiar.

No Quadro 2 mostra a descrição dos artigos selecionados. 
Quadro 2. Descrição dos artigos que abordam os sentimentos dos cuidadores/familiares de pacientes com AVE, segundo título, base de dados, ano, método e sentimentos e dificuldades dos cuidadores.

\begin{tabular}{|c|c|c|c|c|}
\hline Título do artigo & Base de dados & Ano & Método & $\begin{array}{c}\text { Sentimentos e dificuldades que o } \\
\text { cuidador/familiar de pacientes do } \\
\text { AVE apresentam }\end{array}$ \\
\hline $\begin{array}{l}\text { Acidente Vascular Cerebral: } \\
\text { importância do conhecimento } \\
\text { para cuidadores após a alta } \\
\text { Bonelli, M. A., et al }\end{array}$ & BDENF & 2014 & Quantitativo & $\begin{array}{c}\text { Quanto maior o grau de } \\
\text { dependência maior o desgaste } \\
\text { físico; } \\
\text { Falta de conhecimento sobre a } \\
\text { doença e como cuidar é um fator } \\
\text { estressante e de insegurança; } \\
\text { Depressão, sobrecarga físico, } \\
\text { cognitivo e emocional }\end{array}$ \\
\hline $\begin{array}{c}\text { Qualidade de vida de } \\
\text { cuidadores de indivíduos com } \\
\text { acidente vascular encefálico: } \\
\text { associação com características } \\
\text { e sobrecarga } \\
\text { Costa, T. F., et al }\end{array}$ & MEDLINE & 2015 & Quantitativo & $\begin{array}{c}\text { Depressão, sobrecarga físico, } \\
\text { cognitivo, emocional e falta de } \\
\text { recursos financeiros; } \\
\text { Quanto maior o grau de } \\
\text { dependência maior o desgaste } \\
\text { físico, levando a sentimentos de } \\
\text { angústia e stress }\end{array}$ \\
\hline $\begin{array}{l}\text { Sobrecarga de cuidadores } \\
\text { familiares e independência } \\
\text { funcional de pacientes pós- } \\
\text { acidente vascular encefálico } \\
\text { Araújo, J. B., et al }\end{array}$ & LILACS & 2016 & Quantitativo & $\begin{array}{c}\text { Depressão, sobrecarga físico, } \\
\text { cognitivo, emocional e falta de } \\
\text { recursos financeiros; } \\
\text { Aprisionamento e isolamento da } \\
\text { vida social, desencadeia depressão } \\
\text { nos cuidadores }\end{array}$ \\
\hline $\begin{array}{l}\text { Sobrecarga de cuidadores } \\
\text { familiares de idosos com } \\
\text { acidente vascular encefálico } \\
\text { Costa, T. F., et al }\end{array}$ & LILACS & 2015 & Quantitativo & $\begin{array}{l}\text { Falta de conhecimento sobre a } \\
\text { doença e como cuidar é um fator } \\
\text { estressante e de insegurança; } \\
\text { Medo, culpa e ansiedade; } \\
\text { Depressão, sobrecarga físico, } \\
\text { cognitivo, emocional e falta de } \\
\text { recursos financeiros; } \\
\text { Aprisionamento e isolamento da } \\
\text { vida social, desencadeia depressão } \\
\text { nos cuidadores }\end{array}$ \\
\hline $\begin{array}{l}\text { Nursing home care } \\
\text { educational intervention for } \\
\text { family caregivers of older } \\
\text { adults post stroke (SHARE): } \\
\text { study protocol for a } \\
\text { randomized trial. } \\
\text { Day, C. B., et al }\end{array}$ & MEDLINE & 2018 & Quantitativo & $\begin{array}{c}\text { Depressão, sobrecarga físico, } \\
\text { cognitivo, emocional e falta de } \\
\text { recursos financeiros; } \\
\text { Falta de conhecimento sobre a } \\
\text { doença e como cuidar é um fator } \\
\text { estressante e de insegurança; } \\
\text { Quanto maior o grau de } \\
\text { dependência maior o desgaste físico }\end{array}$ \\
\hline $\begin{array}{l}\text { Acidente Vascular Encefálico: } \\
\text { características do paciente e } \\
\text { qualidade de vida dos } \\
\text { cuidadores } \\
\text { Costa, T. F., et al }\end{array}$ & MEDLINE & 2016 & Quantitativo & $\begin{array}{l}\text { Esgotamento emocional e físico; } \\
\text { Falta de conhecimento sobre a } \\
\text { doença e como cuidar é um fator } \\
\text { estressante e de insegurança; } \\
\text { Depressão, sobrecarga físico, } \\
\text { cognitivo, emocional e falta de } \\
\text { recursos financeiros }\end{array}$ \\
\hline
\end{tabular}

Fontes: Autores (2022).

A partir da leitura dos principais achados dos artigos selecionados, a abordagem central destes focava na descrição dos sentimentos dos cuidadores/familiares dos pacientes vítimas do AVE. 


\section{Discussão}

Foi evidenciado nos estudos que quanto maior o grau de dependência maior é o desgaste físico, pois a pessoa acometida por AVE torna-se dependente, até para fazer algo simples da sua rotina de vida diária como: pentear o cabelo, escovar os dentes, levar a comida até a boca, tomar banho, se locomover, troca do vestuário, fechar botões, calçar meia e sapatos, pois todas exigem coordenação motora, e o AVE geralmente compromete essa parte, necessitando de uma pessoa para realizar estas atividades por ele (Bonelli et al., 2014 \& Costa et al., 2016).

A literatura afirma que as atividades de cuidado desenvolvidas pelos cuidadores de pacientes com sequelas de AVE trazem grande impacto na vida do paciente e do cuidador. São tarefas rotineiras que exige esforço físico, como: manusear o paciente acamado sem técnicas específicas que traz desgaste a coluna cervical e lombar, desencadeando dor e tensões musculares para o cuidador, além de ser um risco de lesão também para o paciente com AVE, que é movimentado sem cuidados específicos (Bonelli et al., 2014, Costa et al., 2015)

Outro impacto negativo para os cuidadores/familiares mais jovens é que uma elevada parcela deles acaba deixando sua vida profissional e social em um segundo plano, e passam a cuidar exclusivamente do familiar acometido por AVE. No qual esse quadro de dependência e sobrecarrega de quem cuida, acarreta desgaste físico e emocional (Costa et al., 2015).

Além da sobrecarga física, cognitiva e emocional, existe um fator agregante nesses impactos, que é a falta de recursos financeiros, pois cuidar do paciente com AVE gera um sentimento de depressão nos cuidadores, visto que, segundo Costa et al. (2016) esses cuidadores abdicam de suas casas, vida social e profissional, para prestar cuidado a esse familiar que nesse momento necessita de sua ajuda e ao se encontrarem sozinhos com o paciente com o AVE com pouco auxílio de uma equipe multiprofissional, sem os suprimentos hospitalares e sem ajuda na prestação de cuidado por não ter condições de pagar uma pessoa para ajudar, leva-os ao isolamento e desgastes físico, cognitivo e emocional (Costa et al., 2016)

Sobre as condições financeiras, geralmente está agregada a baixa escolaridade do indivíduo vítimas de AVE e de seus familiares, desta forma sobrecarregando o familiar cuidador, dado que, prestar cuidados a outros requer o suprimento de necessidade básica, tais como: alimentação e aquisição de medicamentos. Para Loureiro et al. (2013) a limitação destes recursos financeiros pode traduzir em fator gerador em forma de depressão e estresse ao cuidador.

O estudo de Brito et al. (2008) mostrou que um dos sentimentos negativos é a culpa que é gerada pela "obrigação" que o familiar tem com o sequelado. A culpa para a psicologia é uma emoção penosa resultante de um conflito. E ter um paciente sequelado de AVE muda quase que radicalmente a vida do cuidador/familiar, ele fica a se perguntar buscando resposta para aquela situação, porque o meu familiar? O que eu fiz para passar por isto? E a sensação de que tudo depende dele e por não ter o cuidado devido com aquele familiar, acabou acontecendo o AVE (Brito et al., 2008).

Percebeu-se através dos estudos que a falta de conhecimento sobre a doença e como cuidar é um fator estressante e de insegurança. $\mathrm{O}$ cuidador que na maioria das vezes é um familiar próximo, de repente se depara com um quadro novo, uma pessoa saudável agora está incapacitada, precisando de um cuidado mais especial (Bonelli et al., 2014). Verificou-se que cuidadores com mais informação sobre a doença, apresentaram maior segurança no cuidado (Costa et al., 2015).

Uma vez que o cuidador domine as ações necessárias na promoção do cuidado, este terá mais facilidade e segurança no planejamento cotidiano. É papel dos profissionais de saúde estarem atentos à fragilidade de quem cuida de um paciente com AVE, tendo em vista que esses sujeitos apresentam-se envolvidos em um sentimento de insegurança (Costa et al., 2015 \& Araújo et al., 2016).

Quando os pacientes recebem alta hospitalar e volta para o ambiente familiar, ele pode apresentar algumas condições especiais, como uso de sondas para a alimentação pelo fato que muitos pacientes que sofreram AVE apresentarem dificuldade em deglutir, a sua locomoção pode está restrita ao leito que leva a outras complicações como: prisão de ventre, movimentos peristálticos prejudicados e úlcera por pressão, esse acaba necessitando de um cuidador com conhecimento para acatar as suas 
necessidades. Porém, diante destas dificuldades o cuidador que não sabe como agir, se sente estressado, com medo e inseguro (Araújo et al. 2016).

$\mathrm{Na}$ alta hospitalar desses pacientes é fundamental que os profissionais de saúde, principalmente a enfermagem, exerçam seu papel de repassar para esses cuidadores/familiares como se adaptar ao novo cenário que o seu parente se encontra. Porém, a literatura relata que esta capacitação ao familiar não está sendo realizada gerando sentimentos negativos nesses cuidadores (Costa et al., 2015 \& Araújo et al. 2016). Além disso, é imprescindível que após a alta, as equipes de atenção básica e atenção domiciliar também atuem nesse processo de capacitação dos cuidadores/familiares, além de auxil

Para Ferreira et al. (2011) a dependência de um familiar reflete uma situação de crise que desencadeia estresse, que ameaça o equilíbrio cotidiano do seio familiar. Podendo surgir um desenvolvimento organizacional, psicológico e social, que frequentemente vem acompanhado de sentimentos negativos como medo, culpa e ansiedade (Ferreira et al., 2011).

Ressalta-se que esses sentimentos negativos e prejuízos à saúde do cuidador/familiar, podem ser amenizados e/ou prevenidos se esses tiverem orientação sobre o cuidado, descanso e cuidados com a própria saúde física e mental. Para isto, necessitam de ajuda de outros familiares para o revezamento dos cuidados a fim de lidar com os aspectos específicos da patologia do paciente e do seu grau de dependência, além de profissionais de saúde que os orientem (Costa et al., 2015).

\section{Considerações Finais}

O estudo mostrou que os sentimentos e emoções dos cuidadores com AVE estão relacionados ao desgaste físico, a falta de conhecimento sobre a doença, e como cuidar é um fator estressante, gera insegurança, podendo levar a quadros de depressão, sobrecarga física, cognitiva e emocional. O fato de que em muitos casos o cuidador/familiar precisa abandonar o seu trabalho leva consequentemente a uma diminuição de recurso financeiro para o ambiente familiar, e essa falta de recursos financeiros gera aprisionamento e isolamento da vida social, desencadeando medo, culpa e ansiedade.

Conclui-se então que o cuidador/familiar podem apresentar sentimentos negativos, tais como: ansiedade, depressão, culpa, medo, angústia e solidão, que podem interferir no estado de saúde destes, e sendo assim esse cuidadores precisam de auxílio para que o ato de cuidar não interfira na sua vida social, profissional, e principalmente na sua saúde.

O estudo apresenta uma limitação por se tratar de dados secundários. Sendo assim, os dados apresentados mostram a importância do estudo sobre essa temática, contudo a uma necessidade na elaboração de estudos mais aprofundados, que além das condições emocionais do cuidador/familiar possa abranger como essa condição afeta o seu convívio social e ambiente familiar.

\section{Referências}

Araújo, J. B., Cirne, G. M. B., Lima, N. M. F. V., Cavalcant,i F. A. V., Cacho, E. W. A., \& Cacho, R. O., (2016). Sobrecarga de cuidadores familiares e independência funcional de pacientes pós-acidente vascular encefálico. Rev. Ciênc. Méd. 2016 ; 25(3):107-113. https://pesquisa.bvsalud.org/portal/resource/pt/biblio-859878

Bonelli, M. A., Massa, A. M., Almeida, C. L. \& Calliari, J. S., (2014). Acidente vascular cerebral: importância do conhecimento para cuidadores após a alta hospitalar CuidArte enf 2014; 8(1):16-23. http://fundacaopadrealbino.org.br/facfipa/ner/pdf/cuidarte_enfermagem_v8_n1_jan_jun_2014.pdf

Brevidelli, M. M., \& Domenico, E. B., (2008). Trabalho de conclusão de curso: guia prático para docentes e alunos da área da saúde. Latria

Brito, E. S., \& Rabinovich, E. P., (2008). Desarrumou tudo!O impacto do acidente vascular encefálico na família. Rev saúde soc 2008; 17(2):153-169. https://doi.org/10.1590/S0104-12902008000200015

Cavalcanti, M. C. V., Lamy, Z. V., Filho, F. L., França, A. K. T. C., Santos, A. M., Thomaz, E. B. A. F., Silva, A. A. M., \& Salgado Filho, N., (2013). Fatores associados à qualidade de vida de adultos em hemodiálise em uma cidade do nordeste do Brasil. J Bras Nefrol. 2013; 35(2):79-86. https://doi.org/10.5935/01012800.20130014

Cameron, J. I., O’Connell, C., Foley, N., Salter, K., Booth, R., Boyle, R., Cheung, D., Cooper, N., Corriveau, H., Dowlatshahi, D., Dulude, A., Flaherty, P., Glasser, E., Gubitz, G., Hebert, D., Holzmann, J., Hurteau, P., Lamy, E., LeClaire, S., \& Lindsay, P. (2016). Canadian Stroke Best Practice Recommendations: Managing transitions of care following Stroke, Guidelines Update $2016 . \quad$ International Journal of Stroke, 11(7), 807-822. https://doi.org/10.1177/1747493016660102 
Research, Society and Development, v. 11, n. 3, e6811326280, 2022

(CC BY 4.0) | ISSN 2525-3409 | DOI: http://dx.doi.org/10.33448/rsd-v11i3.26280

Costa, T. F., Costa, K. N. F. M., Martins, K. P., Fernandes, M. G. M., \& Brito, S. S., (2015). Sobrecarga en los cuidadores familiares de ancianos accidente cerebrovascular. Esc Anna Nery 2015; 19(2):350-355. https://doi.org/10.5935/1414-8145.20150048

Costa, T. F., Gomes, T. M., Viana, L. R. C., Martins, K. P., \& Costa, K. N. F. M., (2016). Acidente vascular encefálico: características do paciente e qualidade de vida de cuidadores. Rev Bras Enferm 2016; 69(5):933-9. https://doi.org/10.1590/0034-7167-2015-0064

Costa, T. F., Costa, K. N. F. M., Fernandes, M. G. M., Martins, K. P., \& Brito, S. S., (2015). Qualidade de vida de cuidadores de indivíduos com acidente vascular encefálico: associação com características e sobrecarga. Rev Esc Enferm USP - 2015; 49(2):245-252. https://doi.org/10.1590/S0080623420150000200009

Costa, G. V. S., \& Romeo, A. L. B., (2021). Perfil epidemiológico dos óbitos por acidente vascular encefálico no Brasil entre 2007 e 2016 : um estudo de base de dados nacional. Revista De Medicina, 100(4), 335-342. https://doi.org/10.11606/issn.1679-9836.v100i4p335-342

Day, C. B., Bierhals, C., Santos, N., Mocellin, D., Predebon, M. L., Dal Pizzol, F., \& Paskulin, L. (2018). Nursing home care educational intervention for family caregivers of older adults post stroke (SHARE): study protocol for a randomised trial. Trials, 19(1), 96. https://doi.org/10.1186/s13063-018-2454-5

Falcão, I. V., Carvalho, E. M. F., Barreto, K. M. L., Lessa, F. J. D., \& Leite, V. M. M., (2004). Acidente vascular cerebral precoce: implicações para adultos em idade produtiva atendidos pelo Sistema Único de Saúde. Revista Brasileira de Saúde Materno Infantil [online]. 2004, v. 4, n. 1 pp. 95-101. https://doi.org/10.1590/S1519-38292004000100009

Ferreira, C. G., Alexandre, T. S., \& Lemos, N. D., (2011). Fatores associados à qualidade de vida de cuidadores de idosos em assistência domiciliária. Saúde Soc. São Paulo 2011; 20(2):398-409. https://doi.org/10.1590/S0104-12902011000200012

Floriano, L. A., Azevedo, R. C. S., Reiners, A. A. O., \& Sudre, M. R. S., (2012). El cuidado brindado por el familiar cuidador de ancianos dependientes en el hogar en el contexto de la estrategia de Salud de la Familia. Texto \& Contexto - Enfermagem [online]. 2012, v. 21, n. 3, pp. 543-548. https://doi.org/10.1590/S0104-07072012000300008

Hoepers, N. J., Birolo, I. V. B., Cerreta, L. B., Soratto, M. T., \& Machado, A., (2019). Atuação da equipe de Estratégia Saúde da Família no domicílio de pacientes com acidente vascular encefálico. Enfermagem Brasil Setembro / Outubro 2013;12(5). https://doi.org/10.33233/eb.v12i5.3765

IBGE (2018). Número de idosos cresce 18\% em 5 anos e ultrapassa 30 milhões em 2017. https://agenciadenoticias.ibge.gov.br/agencia-noticias/2012-agenciade-noticias/noticias/20980-numero-de-idosos-cresce-18-em-5-anos-e-ultrapassa-30-milhoes-em-2017

Israel, N. E. N., Andrade, O. G., \& Teixeira, J. J. V., (2011). A percepção do cuidador familiar sobre a recuperação física do idoso em condição de incapacidade funcional. Ciência \& Saúde Coletiva [online]. 2011, v. 16, suppl 1, pp. 1349-1356. https://doi.org/10.1590/S1413-81232011000700069

Loureiro, L. S. N., Fernandes, M.G.M., Marques, S., Nóbrega, M. M. L., \& Rodrigues R. A. P. Sobrecarga de los cuidadores familiares del adulto mayor: prevalencia y asociación con las características del adulto mayor y del cuidador. Rev. Esc. Enferm. USP 2013; 47(5):1133-40. https://doi.org/10.1590/S0080623420130000500017

Mendes, K. D. S., Silveira, R. C. C. P., \& Galvão, C. M., (2008). Revisão integrativa: método de pesquisa para a incorporação de evidências na saúde e na enfermagem. Texto Contexto Enferm, Florianópolis, 2008 Out-Dez; 17(4): 758-64. https://doi.org/10.1590/S0104-07072008000400018

Oliveira, B. C., Garanhani, M. L., \& Garanhani, M. R., (2011). Cuidador de persona con accidente cerebro vascular: necesidades, sentimientos y orientaciones recibidas. Acta Paul Enferm 2011; 24(1):43-49. https://doi.org/10.1590/S0103-21002011000100006

Pereira, R. A., Santos, E. B. dos, Fhon, J. R. S., Marques, S., \& Rodrigues, R. A. P. (2013). Burden on caregivers of elderly victims of cerebrovascular accident. Revista Da Escola De Enfermagem Da USP, 47(1), 185-192. https://doi.org/10.1590/S0080-62342013000100023

Smeltzer, S. C., \& Bare, B. G., (2005). Tratado de Enfermagem Médico-cirúrgica.

Sousa, L. M. M., Marques-Vieira, C. M. A., Severino, S. S. P., \& Antunes, A. V., (2017). A metodologia de revisão integrativa da literatura em enfermagem. Revista investigação em enfermagem - novembro 2017: 17-26. http://www.sinaisvitais.pt/images/stories/Rie/RIE21.pdf\#page=17

Souza, M. T., Silva, M. D., \& Carvalho, R., (2010). Revisão integrativa: o que é e como fazer. Einstein (São Paulo) [online]. 2010, v. 8, n. 1, pp. 102-106. https://doi.org/10.1590/S1679-45082010RW1134

Vieira, C. P. B., Fialho, A. V. M.. \& Moreira, T. M. M., (2011). La producción científica de la enfermería Brasileña sobre el cuidador informal de ancianos, 1979 a 2007. Texto Contexto Enferm, Florianópolis, 2011 Jan-Mar; 20(1): 160-6. https://doi.org/10.1590/S0104-07072011000100019 